\title{
The Study of Regional Structures Identi- fication by Using Remote Sensing Images in Metallogenic Deposit Prognosis
}

\author{
Dongdai Zhou ${ }^{1,}$, Wei Zhao ${ }^{2}$, Shaochun Zhong ${ }^{1}$, Sunsun Li $^{1}$ \\ ${ }^{1}$ School of Software, Northeast Normal University, Changchun, China \\ 130024,ddzhou@nenu.edu.cn sczhong@nenu.edu.cn \\ ${ }^{2}$ School of Computer, Harbin Institute of Technology, Harbin, China, 150001 \\ ${ }^{3}$ China University of Geosciences, Wuhan, China 430074
}

\begin{abstract}
As an effective assistant method, remote sensing technology has been used in metallogenic deposit prognosis for many years. One key application is to identify regional structures by using remote sensing images. However, there is not a very effective way to do so yet. This paper presents a regional structures identification model by using remote sensing images, and the method of automatic extracting feature information from remotesensing images, and recognizing regional structures through spatial correlative analysis with gravitation, magnetic information, which includes remote sensing data processing, linear and circular structures extraction, and spatial correlative analysis of gravitation, magnetic and remote sensing information.
\end{abstract}

Keywords: remote-sensing, image processing, feature extraction, regional structure

\section{Introduction}

The remote sensing image reflects the ground object's radiation. In the process of analyzing of the geologic anomaly, the remote sensing image plays an important role in metallogenic deposit prognosis.
The general method of using remote sensing technique to predict and assessment metallogenic deposit can be generalized in the following three aspects ${ }^{[1-7]}$ :

(1) Extract the features of geologic body from the remote sensing data to identify the regional structures (linear structures, circular structures);

(2) Analysis of the stratum, rock type;

(3) Analysis of the alteration type and mineral content.

Among them, how to extract the remote sensing feature information to identify regional structures is the key. In recent ten years, although a great deal of work has been done ${ }^{[8-13]}$, the main methods to identify regional structure are manual and do not integrating with other information (such as gravitation, magnetic information). Because of this shortcoming, the application of remote sensing technique in metallogenic deposit prognosis has been limited.

In this paper, we are engaged in solving this problem via building a model of identifying regional structures by using remote-sensing images, and the approach of automatic extracting feature information from remote-sensing images, and identifying regional structures through spatial correlative analysis with gravitation, magnetic information. Section 2 introduces the model of regional structures identification. Section 3 specifies the me-

Proceedings of the 11th Joint Conference on Information Sciences (2008)

Published by Atlantis Press

(C) the authors 
thod of automatic extracting feature information from remote-sensing images, and the approach to identify regional structures through spatial correlative analysis with gravitation, magnetic information. Section 4 specifies a case study of our model and method. Finally, the paper is concluded in Section 5.

\section{The model of regional structures identification by using remote sens- ing image in metallogenic deposit prognosis}

In the process of metallogenic deposit prognosis by using remote sensing spatial information, it is very important to choose rational image features and processing methods according to specified regional geologic environment and application purposes ${ }^{[3]}$. In order to extract remote sensing image linear, circular features automatically, there are many threshold values need to be predefined ${ }^{[13-}$ 15] through both the analysis of application purpose and the expert's experiential knowledge. Otherwise, there will be quite differences between the extracting results and practical geologic structure.

Our model of regional structures identification by using remote sensing images in metallogenic deposit prognosis includes two key components: one is knowledge database, another is inference engine. Knowledge database is the aggregation of knowledge and experience concerning remote sensing image, it not only includes the geologic body's own knowledge, but also includes experts' experiences and methods of the remote sensing information interpretation. The inference engine mainly includes digital remote sensing images disposal and analysis algorithm, and the strategy of application those algorithms. It performs the regional structures extraction based on the knowledge database. The architecture of the model of regional structures identification by using remote sensing image is showed in Fig. 1.

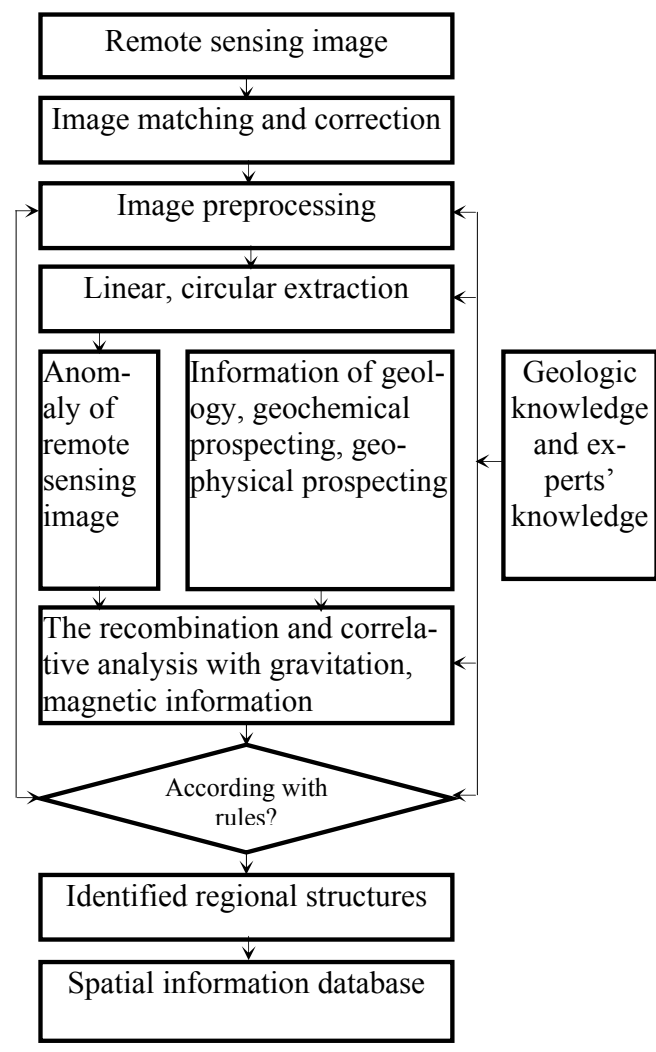

Fig. 1 The architecture of the model of regional structures identification

\section{The methods of automatic extract- ing remote sensing features and re- gional structures identification}

The methods of automatic extracting remote sensing features and regional structures identification involve three key approaches as following:

(1) Extracting remote sensing linear features automatically.

(2) Extracting remote sensing circular features automatically.

(3) Spatial correlative analysis of gravity, magnetism, and remote sensing information. 


\subsection{The automatic extraction ap- proach of remote sensing linear features}

(1) Smoothed filter preprocess. It is to apply the edge holding filter to wipe off the noise in the remote sensing images without blurring their edges. The edge holding algorithm includes the following steps:

(1) Make a proper adjacent domain for every pixel point of the gray level image $(i, j)$, and respectively calculate the gray distributed average degree $V$ of top left corner sub adjacent domain, bottom left corner sub adjacent domain, upper right corner sub adjacent domain, bottom right sub adjacent domain of every pixel point $(i, j)$.

(2) Take the average value that the least average degree as the pixel point's new grey level value.

$V=\sum f^{2}(i, j) \ldots\left[\sum f(i, j)\right]^{2} / N$

(2) Edge extracting. In general, geological structure is the structural line which is certain continuity. Therefore it is sound to extract linear body by adopting the edge tracing algorithm. The edge tracing algorithm is depicted as follow:

(1) Launch raster scan to every point in image, respectively judge whether the differential of 16 directions (from $0^{\circ}$ to $180^{\circ}$ ) is bigger than the threshold value $\mathrm{T}$. If the differential of a point in a direction is bigger than the threshold value $\mathrm{T}$, this point is regarded as the origin point, and begin tracing along this point.

(2) Judge the 16 directions of next point of this direction, and find out the biggest differential direction, then judge whether it is bigger than the threshold value $T$. If it is bigger, loop this step. Else judge whether the tracing length is bigger than length threshold value $L$. If it is bigger, then output the tracked edge image. Else give up this direction tracking of this tracking origin point.

(3) The automatic extraction of linear body. The Hough transformation based on the vote principle can map image from image space to parameter space. In Hough transformation, the parameter space is generally designed into an accumulator array which expresses the discrete parameter values. According to the transformation equation, every point on straight line in image space can vote some parameter combination, while the peak value of parameter space is just the parameter that expresses this curve. In this way, the linear body extraction problem in edge image can be transformed into the problem of seeking the peak value in the parameter space. The linear body extraction algorithm based on Hough transformation is depicted as follows:

(1)To rotate the original image to perform the coordinate transformation from image space to parameter space. The transformation equation is:

$$
\begin{aligned}
& x^{\prime}=x \cos \theta+y \sin \theta \\
& y^{\prime}=-x \sin \theta+y \cos \theta
\end{aligned}
$$

where $\mathrm{x}, \mathrm{y}=$ the original image spatial coordinates $x^{\prime}, y^{\prime}=$ the parameter spatial coordinates.

(2)To quantize the parameter space $\left(x^{\prime}, y^{\prime}\right)$, the quantized interval is $\left(\Delta x^{\prime}, \Delta y^{\prime}\right)$; Suppose that every unit in parameter space is an accumulator, set the initial value of the accumulator array $A\left(x^{\prime}, y^{\prime}\right)$ zero.

(3) To Add 1 to the accumulator where every point $(\mathrm{x}, \mathrm{y})$ in space meets parameter equation.

(4) To scan the accumulator array $A\left(x^{\prime}, y^{\prime}\right)$ on the horizon and vertical ori- 
entation, if the value of the array meta is bigger than threshold value $T_{v}$, this array meta is deemed to be a linear entity. If the interval among several linear entities is smaller than threshold value $T_{v}$, these linear entities constitute "line segment". The first and last linear entities are respectively the origin point and end point of the line segment.

(5) To carry out the transformation from parameter space to original image space

$$
\begin{aligned}
& x=x^{\prime} \cos \theta-y^{\prime} \sin \theta \\
& y=x^{\prime} \sin \theta+y^{\prime} \cos \theta
\end{aligned}
$$

where $x, y=$ the original image spatial coordinate, $x$ ', $y^{\prime}=$ the parameter spatial coordinate.

6) To connect line segment. Since the linear structure has intermittent character in image, it is rational to regard the line segments which have similar direction and vicinal distance as the same linear structure, and connect them. The determine condition of the connection is:

$$
\Delta d=\left|d_{2}-d_{1}\right|<T_{1}
$$

where $d_{1}=\sqrt{\left(x_{0}-x_{1}\right)^{2}+\left(y_{0}-y_{1}\right)^{2}}$

$$
d_{2}=\sqrt{\left(x_{0}-x_{2}\right)^{2}+\left(y_{0}-y_{2}\right)^{2}}
$$

$\mathrm{x}_{0}, \mathrm{y}_{0}=$ the coordinate of center point of image

$\mathrm{x}_{1}, \mathrm{y}_{1}, \mathrm{x}_{2}, \mathrm{y}_{2}=$ respectively the coordinate of the center point of the two line segments

$\mathrm{T}_{1}=$ the predeterminate distance difference threshold value.

$d_{12}=\sqrt{\left(x_{2}-x_{1}\right)^{2}+\left(y_{2}-y_{1}\right)^{2}}<T_{d}$

where $T_{d}=$ the predeterminate distance threshold value between the center point of the two line segment.

$$
\Delta \alpha=\left|\alpha_{2}-\alpha_{1}\right|<T_{a}
$$

where $T_{\alpha}=$ the pre-determinate angle difference threshold value.

$\alpha_{1}, \alpha_{2}=$ respectively the obliquity of the two line segments.

\subsection{The automatic extraction ap- proach of remote sensing image circular features}

\subsubsection{The preprocessing of smoothed filter}

The method is the same with the one in linear extraction basically. In order to enhance the image edge, it also can use second differential convolution die plate and Gaussian filter during the image preprocessing.

\subsubsection{Automatic circular extraction}

We adopt two algorithms to extract circular feature. One is Canny edge detection, the other is Fourier descriptors.

(1) Canny edge detection ${ }^{[14]}$. The general idea of edge detection is to find out the pixel point with local maximum gradient amplitude value in image. But the edge detection algorithm has conflict between choking back noise and locating edges better. Canny edge detection is the first derivative of Gaussian function. It has robustness to noise and better localization of edges. The algorithm is as follows:

(1) Smooth the image with the Gaussian filter;

(2) Calculate the amplitude value and direction of the gradient by applying the finite-difference of first order partial derivative;

(3) Put up non-maximum suppression on the gradient amplitude value;

(4) Detect and connect the edge by using the double threshold value algorithm.

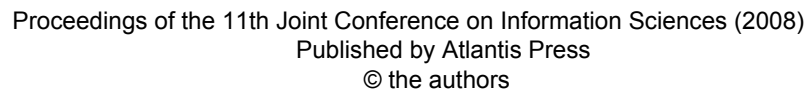


(2) Perform boundary approximation by using the Fourier descriptors. The position function along with the closure contour is periodic, so Fourier series can be used to approach the contour. The resolution of contour approximation is determined by the number of item of Fourier series. The boundary of closure object can be represented by a single dimension coordinate sequence $u_{n}$ :

$u_{n}=x(n)+i y(n), \mathrm{n}=0,1,2, \ldots, N-1$

The discrete Fourier transform of $u_{n}$ is defined as:

$$
\begin{gathered}
\boldsymbol{U}_{\mathrm{n}}=\sum_{k=0}^{N-1} a(k) e^{\frac{i 2 n k n}{N}}, 0 \leq n \leq N-1 \\
a(k)=\frac{1}{N} \sum_{n=0}^{N-1} u(n) e^{\frac{i 2 n k n}{N}}, 0 \leq n \leq N-1
\end{gathered}
$$

Complex coefficient $a(k)$ is called the Fourier descriptors of boundary.

\subsection{Spatial correlative analysis of gravity, magnetism, and remote sensing information}

In order to establish the region structure framework of metallogenic prediction, it is needed to carry out spatial correlative analysis for the remote sensing linear system which reflects the earth's surface information and the gravitational and magnetic linear structures which reflects the deep part, and to establish the structural linear system by studying their correlation. The spatial correlative analysis among remote sensing linear construction and the gravitational, magnetic upward continuation construction mainly includes the two following aspects.

\subsubsection{Knowledge database}

The experts give basic rules that determine the spatial correlation among remote sensing linear body and gravitational, magnetic structures is:
(1)The two lines' morphology and position is close;

(2) The trend of the two lines is close;

(3) If more than one remote sensing linear body have spatial correlation with one of gravitational, magnetic construction line, moreover they are close to each other and have same trend, then these linear bodies belong to the same linear construction.

(4) For each deep linear structure of gravity, magnetism, it must be accompanied with at least one long remote sensing linear body, otherwise, carry out the remote sensing linear extraction again.

\subsubsection{Linear spatial correlative analysis algorithm based on numerical calcula- tion matching}

The Linear spatial correlative analysis algorithm based on numerical calculation matching involves the following steps:

(1) Choose one of the axial lines on the map as one of main lines and mark it with $\mathrm{L}_{1}$, choose another axial line which is accompanied with $\mathrm{L}_{1}$ and mark it with $\mathrm{L}_{2}$, calculate the parallel distance $S$ between line.

(2) Respectively calculate the sum value $\theta_{1}$ and average value $\theta_{2}$ of the gradient of each straight segment that is made up of two near points on $\mathrm{L}_{1}$ and $\mathrm{L}_{2}$.

(3) Calculate $L_{1}, L_{2}$ respectively, which are the length of line $\mathrm{L}_{1}$ and $\mathrm{L}_{2}$,

(4) If it satisfy with the following rules: $\left|\theta_{1}-\theta_{2}\right| \leq \beta$, and $\left|L_{1}-L_{2}\right| \leq \gamma$, then $L_{2}$ and $L_{1}$ are interrelated, otherwise, they are not interrelated. Here, $\alpha, \beta$ and $\gamma$ are the predefined thresholds.

\section{Case Study}

By applying our model and approaches presented above, we identify the regional structures of an area called "Da caotan" in the northwest of China. Fig.2 is the TM remote sensing image of this area af-

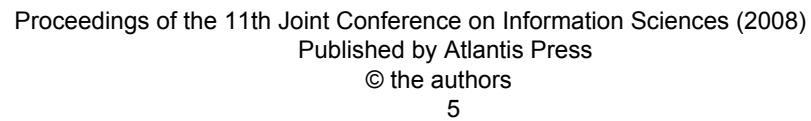


ter image matching and correction. The result of the automatic extraction of remote sensing linear features of Fig. 2 is showed in Fig.3. Fig.4 shows the spatial correlative analysis of gravity, magnetism, and remote sensing linear structures. From Fig.4, we learn that the remote sensing linear structures extracted by our approaches of this experiment area are corresponded with the gravity and magnetism linear structures of this area. It shows that our model and approaches is satisfied in general.

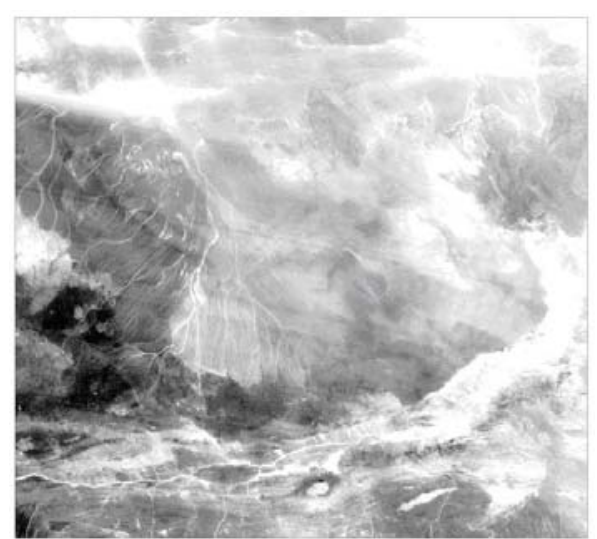

Fig. 2 Da Caotan's TM remote sensing image after image matching and correction

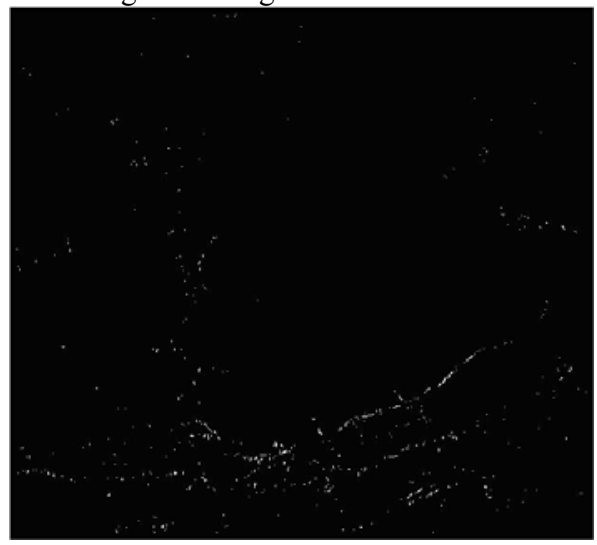

Fig. 3 The extraction result of remote sensing linear features of Fig.2

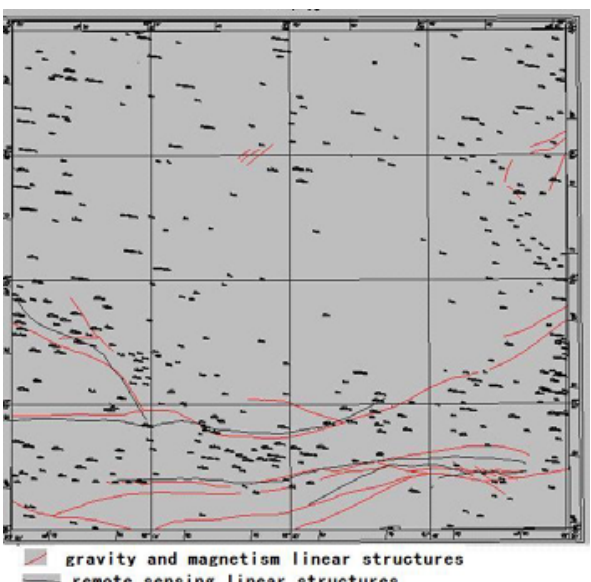

Fig. 4 The spatial correlative analysis of gravity, magnetism, and remote sensing linear structures

\section{Conclusions}

Appling our regional structures identification model and methods, we can automatically extract the linear structures with geologic significance and effectively establish the region structural framework of metallogenic deposit. Furthermore, our experience suggests: (1) the automatic integration of remote sensing spatial information database can only be achieved under the supporting of the database of experts knowledge. (2) The gravitational and magnetic interpretative result which reflects the structures of deep parts is an important standard to validate the result of remote sensing image choosing, filtering preprocessing effect both linear and circular extraction. It is also the basis of establishing the regional structures. (3) Spatial correlative analysis is a sound way for the synthesis integrating of multisource geo-science information.

\section{References}

[1] Ding Jianhua, Xiao Keyan, “The application of remote sensing in mineral resource assessment area", 
PROGRESS IN GEOPHYSICS, VoI.21 No.2, pp588-593, 2006.

[2] Yuxin Ye, Ping Yu, Shi Wang.,et al., "Synthetic information prediction system for crisis mine based on GIS", Global Geology,vol.9, pp94-99,2006

[3] WangShicheng, YeShuisheng, "Theory and Method of Mineral Resource Prediction Based on Synthetic Information", Global Geology,vol.14 No.3, pp207-214,2003.

[4] Feng J., Huang X.Y., "Roles of remote sensing technology on monitoring resources and environment and its trends", Remote Sensing Technology and Application, pp. 59-65,1999.

[5] He G.J., Hu D.Y., Cong B.L., et al., "Information mining and knowledge discovery from satellite remote sensing data based on information theory - a study case on geological application", Remote Sensing Technology and Application, pp. 42-47,1999.

[6] Zhang Y.B., Qu J.H., "Remote sensing technique development and its geological application", Remote Sensing for Land \& Resources, pp. 67-75,1998

[7] Zhao P.D., Wang J.G., "Geologic anomaly of China”. Earth Science Journal of China University of Geosciences, pp. 117-127, 1995.

[8] Chi G.B., LI Y., Ding X., et al., "The study of multidisciplinary prediction system of mineral prospecting based on GIS", Geochimica, pp. 91-99, 1997.

[9] Scott G.J., Edge detection in petrographic images using the rotation polarizer stage, Computer \& Geosciences, pp. 745-751, 1999.

[10] Lee J.W., Kweon I.S., Extraction of line features in a noisy image , Pattern Recognition, pp. 1651-1660, 1997

[11] Bolton A.G., Brown S.F., Moran W., Acomputationally efficient algorithm for enhancing linear features in images, Pattern Recognition, pp. 2017-2025, 1996.

[12] Staib L.H., Duncan J.S., Boundary finding with parametrically deformable models, IEEE Transpattern Analysis and Machine Intelligence, pp. 1061-1075,1992.

[13] Venkatesh R., Automatic lineament extraction from digital images using a segment tracing and rotation transformation approach, Computer \& Geosciences, pp. 555-591, 1995.

[14] Starkey J., Samantaray A.K., Edge detection in petrographic images , Journal of Microscopy, pp. 263-266, 1993.

[15] Gao J.C., Wang G.J., Use of microcomputer in automated lineament detection from remote-sensed imagery $[\mathrm{J}]$. Remote Sensing of Environment, pp. 62-67, 1994.

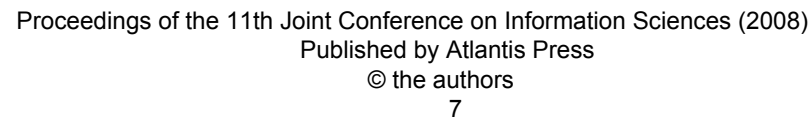

